\title{
Treatment of psychosis in elderly people
}

\author{
Salman Karim \& Eleanor J. Byrne
}

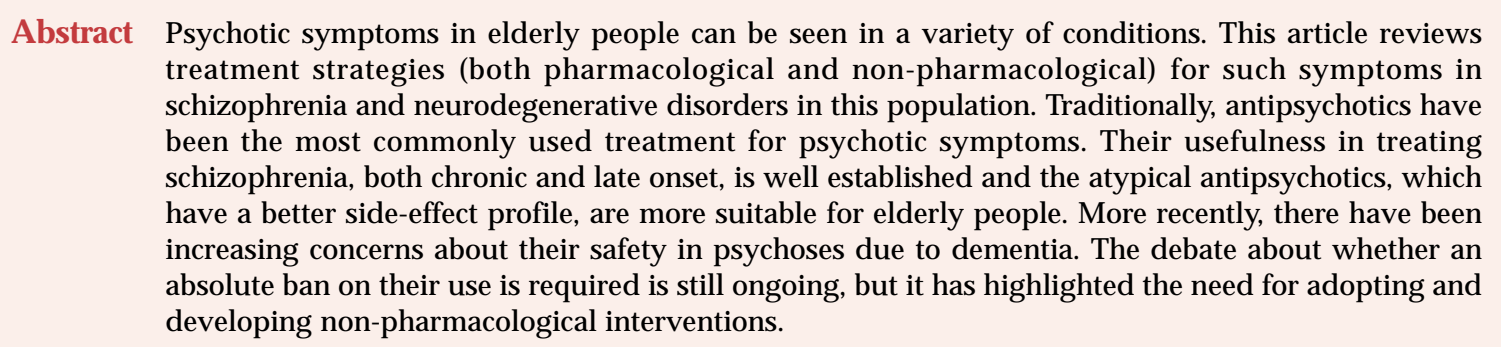

The key feature of psychosis lies in the misinterpretation of the nature of reality, which is reflected in impaired perceptions and interpretation of the environment, false beliefs, and disorganised patterns of speech and behaviour. In clinical practice the word 'psychosis' is commonly used to describe a severe mental illness in which delusions and hallucinations are prominent.

Among elderly patients psychotic symptoms can be seen in a wide range of conditions. The causes and clinical manifestations of the symptoms usually vary with the underlying condition. Psychotic symptoms of acute onset are usually seen in delirium secondary to a medical condition, drug misuse and drug-induced psychosis. Chronic and persistent psychotic symptoms may be due to a primary psychotic disorder (chronic schizophrenia, late-onset schizophrenia, delusional disorders, affective disorders), psychosis owing to neurodegenerative disorders (Alzheimer's disease, vascular dementia, dementia with Lewy bodies and Parkinson's disease) or chronic medical conditions.

Psychotic symptoms are not uncommon in the elderly population and prevalence figures in community samples range from 0.2 to $4.7 \%$ (Targum \& Abbott, 1999). In nursing homes prevalence rates from $10 \%$ to as high as $63 \%$ have been reported (Zayas \& Grossberg, 1998). In a 3-year follow-up study of psychotic symptoms in a population-based sample of very old people (above 85 years of age) without dementia, Östling \& Skoog (2002) reported a prevalence of $7.1-13.7 \%$. They also reported that hallucinations and paranoid ideation were associated with increased incidence of dementia and mortality within 3 years.

Psychotic symptoms can be associated with aggressive or disruptive behaviour (Gilley et al, 1997) and are often a source of distress to caregivers (Zarit et al, 1986; Schneider et al, 1997). They can result in neglect and abuse of elderly patients (Steele et al,1990) and persistent symptoms often result in institutionalisation, which imposes a heavy financial burden (Stern et al, 1997).

A number of factors have been hypothesised to contribute to an increased risk of psychosis in elderly people (Box 1), and the combination of these make its management complicated in older patients.

\section{Box 1 Increased risk of psychosis in elderly people: contributing factors}

- Age-related deterioration of frontal and temporal cortices

- Neurochemical changes associated with aging

- Social isolation

- Sensory deficits

- Cognitive decline

- Age-related pharmacokinetic and pharmacodynamic changes

- Polypharmacy

(Targum \& Abbott, 1999; Targum \& Steven, 2001)

Salman Karim is a lecturer in old age psychiatry at University of Manchester (Division of Psychiatry, Education and Research Centre, Wythenshawe Hospital, Manchester M23 9LT, UK. Tel: 0161291 5867/5884; fax: 0161 291 5882; e-mail: skarim@fs1.with.man.ac.uk). His special interests are schizophrenia in elderly people and the role of inflammation in the aetiology of Alzheimer's disease. Eleanor Byrne is a senior lecturer in old age psychiatry at the University of Manchester with a special interest in dementia with Lewy bodies. 


\section{Use of antipsychotics in elderly people}

Elderly people show variable responses and increased sensitivity to medications in general (Avron \& Gurwitz, 1990) and to antipsychotics in particular. Age-related bodily changes affect the pharmacokinetics and pharmacodynamics of antipsychotic drugs, which have numerous side-effects (Box 2) that can be more persistent and disabling in older people. Tardive dyskinesia, for example, can lead to a number of physical and psychological complications, including difficulty in eating and swallowing, weight loss, falls, difficulty in keeping balance and depression (Jeste, 2004). The risk of developing tardive dyskinesia from typical (older) antipsychotics is 5-6 times higher in older people (Kane, 1999), although recent studies indicate that the newer atypicals may pose a lower risk of this side-effect and may therefore be safer for older people (Jeste, 2004).

\section{Box 2 Potential side-effects of antipsychotics} in elderly people

Extrapyramidal side-effects

- Pseudoparkinsonism

- Akathesia

- Acute dystonia

- Tardive dyskinesia

Anticholinergic effects

- Urinary hesitancy

- Constipation

- Blurred vision

- Dryness of mouth

- Delirium

Postural hypotention

Sedation

Hypersalivation

Gastrointestinal effects

- Nausea

- Constipation

- Diarrhoea

Liver effects

- Cholestatic jaundice

- Raised transaminase enzyme activities

Cardiovascular effects

- ECG abnormalities: QTc prolongation

Endocrine effects

- Weight gain

- Diabetes mellitus

Epilepsy
Antipsychotics can also increase the rate of cognitive decline (Holmes et al, 1997; McShane et al, 1997); they have been associated with neuroleptic sensitivity syndrome, a potentially lethal adverse effect (Byrne et al, 1992; McKeith et al, 1992); and some are now subject to restrictions under the Committee on Safety of Medicines (CSM). Having reviewed the literature on the use of risperidone and olanzapine for the treatment of behavioural and psychological symptoms in dementia, the chairman of the CSM concluded that each was associated with at least a two-fold increase in the risk of stroke and therefore should no longer be used in dementia (Duff, 2004). Herrmann et al (2004), however, found no difference in the risk of stroke between risperidone and olanzapine compared with typical neuroleptics used in the treatment of dementia $(n=11400)$. Others have subsequently commented on the potential detrimental effects of such a blanket ban (Mowat et al, 2004).

Following the CSM restriction on risperidone and olanzapine, a Working Group for the Royal College of Psychiatrists' Faculty of the Psychiatry of Old Age, the Royal College of General Practitioners, the British Geriatrics Society and the Alzheimer's Society also acknowledged a small but significant risk of cerebrovascular adverse events in elderly people, especially in people over 80 years of age, with the use of risperidone and olanzapine (Royal College of Psychiatrists et al, 2004). The Working Group advocated a more balanced approach to their prescription that involves weighing the risks and benefits for individual patients, since these drugs may still be worth using in some circumstances, particularly when alternative drug treatments have similar or worse side-effects and nonpharmacological approaches are not suitable. For other antipsychotics, both typical and atypical, the choice of prescription should be based on the sideeffect profile and risk factors such as cerebrovascular events, postural hypotension and tardive dyskinesia. For people who have been stable on antipsychotics for more than 3 months, cautious withdrawal may be considered. The decision to withdraw or continue should be based on past history and the risks of recurrence. The reasons for using or continuing a particular antipsychotic must be clearly documented and the general practitioner should be involved in the decision-making process.

The discussions about the use and safety of antipsychotics will probably go on for some time, but they have highlighted the need for research on alternative forms of treatment. For the present, it is important to be careful not to do more harm than good when initiating antipsychotic medication for older people and to follow the principle 'start low and go slow' (Zayas \& Grossberg, 2002). 


\section{Schizophrenia}

Older people with schizophrenia have traditionally been divided into two main groups, those who develop the illness in later life and those who have had it from an early age and have now grown old. Historically, it was Kraepelin in the early 20th century who recognised that the non-affective psychosis in young adults that he called 'dementia praecox' could also first become apparent in middle or old age. Bleuler subsequently coined the term 'lateonset schizophrenia' to describe this schizophrenialike illness that arises in old age in the absence of organic brain disease or amnestic syndrome.

There has since been much debate about the nosology and classification of psychotic disorders in old age. Some emphasise the similarities between the early- and late-onset illnesses and others highlight the differences in aetiology, phenomenology and outcome. A consensus on nomenclature was reached in 1998, at a meeting of the International Late Onset Schizophrenia Group (Howard et al, 2000). On the basis of the research evidence on symptoms, family history, brain imaging studies and the nature of the cognitive deficits observed, it was agreed to retain the word schizophrenia for both the early- and late-onset illnesses. However, the late-onset illness was further subdivided into late onset (onset after 40 years of age) and very late onset (onset after 60 years of age). Some comparative features of earlyand late-onset schizophrenia are shown in Box 3, and characteristics of the very-late-onset illness are listed in Box 4.

This classification is, however, not considered final and there is much room for further debate and research. Moreover, there are no separate categories

Box 3 Similarities and differences between early- and late-onset schizophrenia

Similarities

- Genetic risk

- The presence and severity of positive symptoms

- Early psychosocial maladjustments

- Subtle brain abnormalities revealed by imaging

Differences

Late-onset schizophrenia is characterised by:

- fewer negative symptoms

- better neuropsychological performance

- better response to antipsychotics

(Palmer et al, 2001)
Box 4 Characteristic features of very-lateonset schizophrenia

Compared with early- or late-onset schizophrenia, very-late-onset schizophrenia is characterised by:

- associated sensory impairment

- social isolation

- a greater likelihood of visual hallucinations

- a lesser likelihood of formal thought disorder

- a lesser likelihood of affective blunting

- a lesser likelihood of family history of schizophrenia

- a greater risk of developing tardive dyskinesia

- the significantly higher number of females affected than males

(Lisa et al, 2002; Tune \& Salzman, 2003)

for late-onset and very-late-onset schizophrenia in either DSM-IV-TR (American Psychiatric Association, 2000) or ICD-10 (World Health Organization, 1992).

The prevalence of schizophrenia (early, late and very late onset combined) in the population aged 65 years and above is believed to be about $1 \%$ (Cohen et al, 2000). Out of these, nearly $25 \%$ have late- or very-late-onset illness, and the remaining $75 \%$ are people with early-onset schizophrenia who have reached old age (Jeste \& Twamley, 2003).

\section{Pharmacological treatment}

Antipsychotic medications are the most widely used pharmacological treatment for both early- and lateonset schizophrenia in elderly people. Although there is a dearth of well-conducted studies (with few randomised controlled trials), there is some evidence that these drugs improve acute symptoms and prevent relapse (Jeste et al, 1996).

\section{Conventional antipsychotics}

The research literature on the use of conventional antipsychotics in elderly people with schizophrenia is sparse and there are very few recent studies. Significant improvement in psychotic symptoms with the use of haloperidol, trifluoperazine (10$30 \mathrm{mg} /$ day) and thioridazine (40-50 mg/day) was reported in studies carried out in the 1960s (Post, 1966; Tsuang et al, 1971). Thioridazine has since been shown to cause prolongation of the QT interval and its use in elderly people is not recommended.

Depot antipsychotic medication can be useful in elderly patients who have problems adhering to 
medication regimens. Howard \& Levy (1992) reported that low doses of depot antipsychotics (14.4 $\mathrm{mg}$ of flupentixol decanoate or $9 \mathrm{mg}$ of fluphenazine decanoate every 2 weeks) were associated with improved adherence and treatment outcome compared with oral medication.

\section{Atypical antipsychotics}

The newer atypical antipsychotics are currently considered the first-line treatment for older patients owing to their better side-effect profile in comparison with conventional antipsychotics (Tune \& Salzman, 2003). However, limited data are available from controlled trials showing their efficacy and safety in older people.

Clozapine The usefulness of clozapine for treatment-resistant early-onset schizophrenia is well established, but concerns about toxicity and the need for monitoring white cell counts has led to limited use in older patients. A few small studies on its use at lower doses in this population have reported sedation, lethargy and postural hypotension as common side-effects (reviewed by Barak et al, 1999). In their review Barak et al concluded that most showed moderate-to-marked improvement of psychotic features at a relatively low mean dose of $134 \mathrm{mg} /$ day, but cautioned that agranulocytosis may occur more frequently in older people. In light of these risks, clozapine is not a first-line antipsychotic for elderly patients and should probably be used only in cases of treatment resistance and severe tardive dyskinesia (Howard, 2002).

Risperidone and olanzapine Of the atypicals, risperidone is the most extensively studied in the elderly population. It is effective, well tolerated in low doses (1.5-6 mg/day) and produces significant clinical improvement in elderly people with schizophrenia (Katz et al, 1999; Madhusoodanan et al, 1999). Limited data are available on the use of olanzapine in treating older people with schizophrenia. Madhusoodanan et al (1999) compared 151 hospitalised elderly psychiatric patients (mean age 71 years) who received either risperidone or olanzapine. Olanzapine therapy was found to be effective, with side-effects reported in $17 \%$ of the patients, and the authors concluded that the drug was safe and effective in that population. Sajatovic et al (1998) studied olanzapine in an open-label trial with 22 older patients with schizophrenia. They found that it significantly improved symptoms of schizophrenia and had few extrapyramidal sideeffects without adversely affecting comorbid medical problems. Owing to recent concerns about the sideeffects of these two antipsychotics in people with dementia, their use is also likely to be restricted in people with schizophrenia.

Quetiapine On the basis of their review of the literature, Zayas \& Grossberg (2002) have suggested that quetiapine is safe for use in elderly people and is not associated with weight gain. To avoid the common side-effects of postural hypotention, dizziness and agitation, they recommend starting with the lowest possible dose $(25 \mathrm{mg})$ and slowly titrating up to 100-300 mg/day. More recently, Jaskiw et al (2004), in a multicentred open-label trial, have reported safe use in dosages up to $750 \mathrm{mg} /$ day, given in divided doses. As no other study has reported use of quetiapine in such high doses for elderly people, we suspect that only an occasional patient would require a very high dose.

Aripiprazole The latest of the atypical antipsychotics aripiprazole, with its unique mode of action as a partial agonist at $D_{2}$ receptors can be effective in improving both positive and negative symptoms. Furthermore, it is less likely than the other atypicals to cause extrapyramidal symptoms, sedation, weight gain and cardiovascular sideeffects (Hirose et al, 2004). It probably holds promise for both young and older people with schizophrenia, but there are few data on its use, safety and dosing strategies in older people. Madhusoodanan et al (2004) described their clinical experience of aripiprazole in ten elderly people with schizophrenia. They concluded that it is safe, improved both positive and negative symptoms and caused fewer side-effects.

\section{Dosage}

Suggested daily doses of various atypicals for elderly people are given in Table 1 (no data are available for aripiprazole). These should be taken as a guideline only and the dosing regimen should be tailored to the needs of individual patients. The already mentioned strategy of starting low and going slow is probably the safest way of using the newer antipsychotics for which robust safety data are lacking.

Table 1 Recommended doses of atypical antipsychotics for elderly people

$\begin{array}{lcc}\text { Clozapine } & \begin{array}{c}\text { Starting dose } \\ (\mathrm{mg} / \text { day })\end{array} & \begin{array}{c}\text { Maximum dose } \\ (\mathrm{mg} / \text { day })\end{array} \\ \text { Risperidone } & 6.25 & 50-100 \\ \text { Olanzapine } & 0.25-0.5 & 2-3 \\ \text { Quetiapine } & 1-5 & 5-15 \\ \text { Ziprasidone } & 12.5-25 & 100-200 \\ \text { Source: Zayas \& Grossberg (2002). } & 15-20 & 80-160\end{array}$




\section{Electroconvulsive therapy}

Most research on the use of electroconvulsive therapy (ECT) on elderly patients with schizophrenia was conducted during the 1950s and 1960s. Kay \& Roth (1961) reported temporary remission following the use of ECT or neuroleptics in about $25 \%$ of their patients. A better response to ECT in patients with late paraphrenia presenting with prominent affective symptoms was reported by Frost (1969). It appears that, with the introduction of a variety of typical and atypical antipsychotics, the use of ECT on elderly patients with schizophrenia has declined in clinical practice.

\section{Cognitive-behavioural therapy}

The usefulness of cognitive-behavioural techniques in modifying delusional beliefs and controlling hallucinations has been widely reported in younger people (Garety et al, 1994; Fowler et al, 1995). Unfortunately, there have been few attempts to study their use with elderly patients. Agüera-Ortiz et al (1999) have suggested that they might help elderly people gain insight into their illness and provide them with coping strategies to help them live a meaningful life.

McQuaid et al (2000) have developed a novel intervention for older people with schizophrenia that integrates cognitive-behavioural techniques and social skills training. This approach suits the needs of elderly people and aims at reducing their cognitive vulnerabilities and improving their ability to cope with stress and to adhere to other forms of treatment.

\section{Psychosocial therapies}

The effectiveness of psychosocial interventions in improving independent living and social skills in younger people with schizophrenia is well established (Kopelowicz \& Liberman, 1998). Such interventions may also be of importance for elderly patients, a significant number of whom fail to show a complete response to antipsychotics (Howard, 2002). Bartels et al (2004), in a pilot study of elderly people with severe mental illness, found that a combination of interpersonal and independent skills training, together with standard occupational therapy, was associated with improved social functioning and independent living.

\section{Neurodegenerative disorders}

Among the neurodegenerative disorders, psychotic symptoms are commonly seen in Alzheimer's disease, dementia with Lewy bodies and Parkinson's disease. In Alzheimer's disease and Lewy body dementia, psychotic symptoms are thought to be related to the underlying pathophysiology of the condition. In Parkinson's disease, which commonly presents with motor symptoms and dementia, antiParkinsonian medication is the most frequent cause of psychotic symptoms (Mintzer \& Targum, 2003).

\section{Alzheimer's disease}

The prevalence of psychosis in people with Alzheimer's disease ranges between 30 and 50\% (Jeste \& Finkel, 2000). Bassiony et al (2000), in a community-based study of Alzheimer's disease, reported that about one-third of the participants showed evidence of psychotic symptoms and that delusions were more common than hallucinations.

The question of whether delusions in Alzheimer's disease (Box 5) are secondary to the cognitive deficits or are true psychotic phenomena remains unanswered. Hallucinations in Alzheimer's disease can occur in any sensory modality, but visual and auditory hallucinations are the most common (Tariot, 1995). There is some evidence of the association of psychotic symptoms with a rapid decline in cognition in Alzheimer's disease (Förstl et al, 1994; Levy et al, 1999).

\section{Pharmacological treatment of psychosis}

Antipsychotics Antipsychotics have been the most widely used form of treatment for psychosis in Alzheimer's disease (Margallo-Lana et al, 2001), although not without concerns about the safety of their use, as discussed above. A number of fairly recent studies have demonstrated the efficacy of antipsychotics in controlling psychotic symptoms.

Box 5 Four common types of misidentifying delusion seen in individuals with Alzheimer's disease

- The Capgras type The false belief that previously known people (e.g. wife or caregiver) have been replaced by impostors

- The phantom boarder symptom A false belief that guests are living in the person's house

- The mirrorsign The individual misidentifies his or her own mirror image as someone else

- The TV sign Misidentification of television images as real (a variant of this is the magazine sign, in which magazine images on a table are perceived as being real and existing in three-dimentional space (Karim \& Burns, 2003)) 
However, most of these were designed to look at the usefulness of these drugs in controlling the behavioural and psychological symptoms of dementia, not its psychotic symptoms.

Schneider (1996), in a meta-analysis of seven placebo-controlled trials of the use of typical antipsychotics, reported significant but modest efficacy. Devanand et al (1998), in a randomised placebocontrolled dose-comparison trial of haloperidol, reported superior efficacy of doses of 2-3 mg/day, with moderate-to-severe extrapyramidal symptoms occurring in $20 \%$ of patients; a lower dose $(0.5-$ $0.75 \mathrm{mg} /$ day) was no better than placebo.

Of the atypical antipsychotics, there have been a number of randomised placebo-controlled trials of risperidone and olanzapine.

Katz et al (1999), in a randomised double-blind trial comparing risperidone with placebo in nursing-home patients, demonstrated the efficacy of risperidone over placebo; the optimal dose was $1 \mathrm{mg} /$ day. Other studies (De Deyn et al, 1999; Brodaty et al, 2003) have confirmed the efficacy of low doses of risperidone for controlling psychotic symptoms in Alzheimer's disease.

Olanzapine in a dose of $5 \mathrm{mg}$ /day significantly improved psychotic symptoms in Alzheimer's disease in a double-blind placebo-controlled trial of 6 weeks' duration. Higher doses (10 and $15 \mathrm{mg}$ ) showed no added benefit. An open-label follow-up showed that the improvement could be maintained (Street et al, 2000, 2001).

Quetiapine in a dose of 100-300 mg/day has been reported to be well tolerated and to improve psychotic symptoms and hostility in people with Alzheimer's disease (McManus et al, 1999; Tariot et al, 2000; Yeung et al, 2000).

Cholinesterase inhibitors These are routinely used for cognitive deficits in Alzheimer's disease, and more recently their possible usefulness in improving psychotic symptoms has been investigated. Although there have been no prospective double-blind studies, reviews of current data (most studies have been on rivastigmine, donepezil and galantamine) suggest that these drugs are well tolerated and may be of value in preventing or reducing psychotic symptoms in Alzheimer's disease (Finkel, 2004; Wynn \& Cummings, 2004).

\section{Dementia with Lewy bodies}

Dementia with Lewy bodies is probably a part of the spectrum of Lewy body disorders (Byrne, 1997). Its clinical presentation usually varies according to the site of Lewy body formation and associated neuronal pathology. Psychotic symptoms are seen more frequently in Lewy body dementia than in
Alzheimer's disease. Visual hallucinations are the most common symptom and have been reported in up to $80 \%$ of cases; other classic symptoms include fluctuating cognition, Parkinsonian motor symptoms, frequent falls and sensitivity to neuroleptic medication (McKeith et al, 1994). Auditory hallucinations and paranoid delusions are also common, with prevalence rates of $20 \%$ and $65 \%$, respectively (McKeith et al, 1996).

The treatment of psychotic symptoms in Lewy body dementia remains a challenge and most often requires a treatment plan tailored to the characteristics of individual patients. This should strike a balance between use of anti-Parkinsonian medication, which improves motor disorder but may induce psychotic symptoms, or not treating motor symptoms and cautiously treating the psychotic symptoms. This challenge also highlights the importance of nonpharmacological interventions (see below).

\section{Pharmacological treatment}

Antipsychotics People with Lewy body dementia are extremely sensitive to antipsychotics. Small doses can lead to extreme worsening of Parkinsonian symptoms, and about $50 \%$ of individuals experience life-threatening adverse effects (McKeith et al, 1992). Severe reactions may be dose related (Byrne et al, 1992). The above-mentioned adverse effects of neuroleptics in older people have discouraged their use and consequently no robustly designed studies of antipsychotics in Lewy body dementia have been carried out. However, some reports on the use of olanzapine in this population have been published (Walker et al, 1999; Cummings et al, 2002).

Cholinesterase inhibitors A number of studies have reported improvement of psychotic symptoms with the use of cholinesterase inhibitors in Lewy body dementia. A large multicentre double-blind trial comparing rivastigmine with placebo showed significant improvements in delusions and hallucinations (McKeith et al, 2000). Beneficial effects of the use of donepezil have also been reported (Fergusson \& Howard, 2000).

Cholinesterase inhibitors are not yet licensed for the treatment of Lewy body dementia in the UK.

\section{Parkinson's disease}

Psychotic symptoms in Parkinson's disease are most commonly extrinsic (resulting from treatment with anti-Parkinsonian drugs) and only occasionally intrinsic (secondary to the neurodegenerative process involving dopamine-producing cells in other parts of the brain) (Wolters, 2001). Most antiParkinsonian drugs (including levodopa, dopamine 
receptor agonists, dopamine release enhancers such as amantadine, and monoamine oxidase inhibitors such as selegiline) can cause psychotic symptoms.

Between 20 and $60 \%$ of people with Parkinson's disease develop psychotic symptoms (Kuzuhara, 2001; Wolters \& Berendse, 2001). Hallucinations are more frequent than delusions in extrinsic cases (Aarsland et al, 1999) and visual hallucinations are more common than hallucinations in other sensory modalities (Hoeh et al, 2003). Epidemiological studies have found that the risk of psychotic symptoms in Parkinson's disease is higher in later stages of the disease and when there is concurrent dementia or depressive illness (Aarsland et al, 1999; Giladi et al, 2000).

\section{Pharmacological treatment}

Antipsychotics Treatment of psychotic symptoms in Parkinson's disease is difficult owing to older people's sensitivity to antipsychotics in general and to typical antipsychotics in particular. Clozapine has been the most widely used and studied antipsychotic. Several double-blind controlled trials have established its efficacy. The optimal dose to reduce symptoms and minimise side-effects is 6.25-50 mg/day (Hoeh et al, 2003).

There have been several retrospective reports and open-label trials on other atypicals such as risperidone and olanzapine, but none has been shown to improve psychotic symptoms without worsening extrapyramidal symptoms (Breier et al, 2002; Ondo et al, 2002).

Cholinesterase inhibitors There have been encouraging reports on the success of cholinesterase inhibitors such as donepezil and rivastigmine in improving both psychotic symptoms and cognitive deficits in Parkinson's disease (Bergman \& Lerner, 2002; Bullock \& Cameron, 2001; Fabbrini et al, 2002).

\section{Should antipsychotics be used in dementia?}

In addition to general concerns about the saftey of neuroleptics for older people, the use of any medication to treat psychotic symptoms in dementia is increasingly being questioned. Do psychotic symptoms that are not distressing or adversely affecting the patient require treatment with medication (Kidder, 2003)? A careful assessment of potentially remediable environmental causes such as sensory deprivation, poor lighting and social isolation can prevent use of antipsychotics. Addressing other contributory and causal factors such as physical illness and side-effects of medication (Box 6) is equally important.
Box 6 Medications that can cause psychotic symptoms in elderly people during use or on withdrawal

Benzodiazepines

Antihistamines

- Cimetidine

Anti-Parkinsonian drugs

- Levodopa

- Amantadine

- Bromocriptine

- Procyclidine

Anti-arrhythmics

- Digoxin

- Propranolol

- Quinidine

- Procainamide

Anti-inflammatory drugs

- Aspirin

- Indomethacin

Anticonvulsants

- Phenytoin

- Primidone

- Carbamazepine

Steroids

- Prednisolone

Anti-cancer drugs

(Wood et al, 1988;

Targum \& Abbott, 1999; Targum, 2001)

\section{Non-pharmacological treatment of psychotic} symptoms in dementia

Non-pharmacological treatment of behavioural and psychological symptoms (including psychosis) in dementia has been the subject of increasing research in recent years (Overshott et al, 2004). The nonpharmacological approach requires a detailed knowledge of the patient's personality and past psychiatric history, careful listening, observation of the current situation, and effective verbal and nonverbal communication. Cohen-Mansfield (2003) has described a three-stage (Box 7) approach involving assessment, ascertainment of causes of symptoms and planning an intervention such as the following.

Reducing sensory deprivation Practical measures aimed directly at the patient might include a hearing aid or glasses. External measures such as improving lighting, providing enhanced-contrast materials, and larger type faces and objects may also help. An increase in positive stimulation through auditory sensations such as music and tactile sensations such as touch and massage may also prove useful. 
Box 7 The stages of a non-pharmacological approach to symptoms of dementia (Cohen-Mansfield, 2003)

\section{Assessment}

This includes systematic observation of the patient and should concentrate on the following areas:

- identification of the problem through assessment of the symptoms

- assessment of the interaction of symptoms with the environment by dividing them into antecedents and consequences

- clarification of the negative effect of the symptoms, i.e. whether the patient and caregivers are negatively affected by them. This area is important because psychotic symptoms that do not have a negative impact may not require treatment (Kidder, 2003).

Ascertaining possible causes for the symptoms

Some symptoms have environmental causes, and important areas to explore are:

- whether the patient has a negative view of the caregiver

- whether the patient is unable to understand the intentions of caregivers

- whether the patient suffers from social isolation or sensory deprivation

- the patient's misinterpretations of the environment and situations

\section{Planning an intervention}

The following points should be kept in mind:

- the intervention should be tailored to the needs of the particular patient and should address the cause of the symptoms

- the intervention may be directed at the patient, or at the environment, members of staff or the general system of care

- the need for regular assessment and re-evaluation of the intervention, to monitor symptom improvement

Reducing inappropriate inner sensory stimulation Simple practical measures can reduce stimulations that produce psychotic symptoms. Examples include removing mirrors if reflections cause the delusion of having phantom boarders in the house, or drawing a curtains over windows if the patient has a delusion of being spied on or followed.

Measures for specific symptoms Misinterpretation of reality is the basis of a number of psychotic symptoms in dementia. A common symptom such as seeing caregivers as impostors can be addressed by training them to establish a positive relationship with the patient, introduce themselves with each encounter and clearly explain what they are going to do before doing it.

Delusions of infidelity or abandonment in institutionalised patients can be addressed by arranging frequent contact with their families. This can be real or simulated (by using videotapes of family members or simulated presence therapy; Hall \& Hare, 1997; Camberg et al, 1999). Measures such as frequent telephone calls and bringing familiar items from the patient's home can also be helpful in countering feelings of abandonment and betrayal.

The delusion that other people are stealing belongings can be addressed by providing duplicates of items that are easily mislaid (such as reading glasses), providing a remote control finder or using methods such retrieval, which teaches the patient always to return certain items to particular places (McKitrick et al, 1992).

\section{Conclusions}

Antipsychotic drugs commonly and successfully used in younger populations can be prescribed only cautiously for elderly people. Some have been inadequately studied in older age groups and many carry increased risk of dangerous or debilitating side-effects. However, they remain the mainstay of treatment for schizophrenia and related psychotic disorders in elderly people. Cholinesterase inhibitors show some promise for the psychotic symptoms of neurodegenerative disorders, but they have yet to be licensed in the UK to treat all dementias.

Drugs, however, are not the only option for psychotic symptoms, particularly those that neither distress nor endanger the patient. Much can be achieved with non-pharmacological interventions such as environmental changes, sensitive staff training and a patient-centred approach, which offer a safe augmentation of, or even alternative to, medication for a growing population of vulnerable people. 


\section{References}

Aarsland, D., Larsen, J. P., Cummins, J. L., et al (1999) Prevalence and clinical correlates of psychotic symptoms in Parkinson's disease: a community based study. Archives of Neurology, 56, 595-601.

Agüera-Ortiz, L. \& Reneses-Prieto, B. (1999) The place of non-biological treatments. In Late Onset Schizophrenia (eds R. Howard, P. V. Rabins \& D. J. Castle), pp. 50-56. Winchester: Wrightson Biomedical Publishing.

American Psychiatric Association (2000) Diagnostic and Statistical Manual of Mental Disorders (4th edn, text revision) (DSM-IV-TR). Washington, DC: APA

Avron, J. \& Gurwitz, J. (1990) Principles of pharmacology. In Geriatric Medicine (2nd edn) (eds K. Cassel, D. Reisenberg, L. Sorenson, et al), pp. 66-77. New York: Springer-Verlag.

Barak, Y., Wittenberg, N., Naor, S., et al (1999) Clozapine in elderly psychiatric patients: tolerability, safety and efficacy. Comprehensive Psychiatry, 40, 320-325.

Bartels, S. J., Forester, B., Mueser, K. T., et al (2004) Enhanced skills training and health care management for old persons with severe mental illness. Community Mental Health Journal, 40, 75-90.

Bassiony, M. M., Steinberg, M. S., Warren, A., et al (2000) Delusions and hallucinations in Alzheimer's disease: prevalence and clinical correlates. International Journal of Geriatric Psychiatry, 15, 99-107.

Bergman, J. \& Lerner, V. (2002) Successful use of donepezil for the treatment of psychotic symptoms in patients with Parkinson's disease. Clinical Neuropharmacology, 25, 107110.

Breier, A., Sutton, V. K., Feldman, P. D., et al (2002) Olanzapine in the treatment of dopamimetic-induced psychosis in patients with Parkinson's disease. Biological Psychiatry, 52, 438-445.

Brodaty, H., Ames, D., Snowdon, J., et al (2003) A randomised placebo-controlled trial of risperidone for the treatment of aggression, agitation, and psychosis of dementia. Journal of Clinical Psychiatry, 64, 134-143.

Bullock, R. \& Cameron, A. (2001) Rivastigmine for the treatment of dementia and visual hallucinations associated with Parkinson's disease: a case series. Current Medical Research and Opinion, 18, 258-264.

Byrne, J. (1997) Lewy body dementia. Journal of the Royal Society of Medicine, 90 (suppl. 32), 14-15.

Byrne, E. J., Burns, A. \& Waite, J. (1992) Neuroleptic sensitivity in dementia with cortical Lewy bodies. BMJ, 305, 1158-1159.

Camberg, L., Woods, P., Ooi, W. L., et al (1999) Evaluation of simulated presence: a personalized approach to enhance well being in persons with Alzheimer's disease. Journal of the American Geriatrics Society, 47, 446-452.

Cohen, C. I., Cohen, G. D., Blank, K., et al (2000) Schizophrenia and older adults: an overview. Directions for research and policy. American Journal of Geriatric Psychiatry, 8, 19-28.

Cohen-Mansfield, J. (2003) Non-pharmacological interventions for psychotic symptoms in dementia. Journal of Geriatric Psychiatry and Neurology, 16, 219-224.

Cummings, J. L., Street, J., Masterman, D., et al (2002) Efficacy of olanzapine in the treatment of psychosis in dementia with Lewy bodies. Dementia and Geriatric Cognitive Disorders, 13, 67-73.

De Deyn, P. P., Rabheru, K., Rasmussen, A., et al (1999) A randomised trial of risperidone, placebo and haloperidol for behavioural symptoms of dementia. Neurology, 53, 946-955

Devanand, D. P., Marder, K., Michaels, K. S., et al (1998) A randomised, placebo-controlled dose-comparison trial of haloperidol for psychosis and disruptive behaviours in Alzheimer's disease. American Journal of Psychiatry, 155, $1512-1520$.

Duff, G. (2004) Atypical Antipsychotic Drugs and Stroke: Message from Professor Gordon Duff, Chairman, Committee on Safety of
Medicines (CEM/CMO/2004/1). http://medicines.mhra. gov.uk/ourwork/monitorsafequalmed/safetymessages / antipsystroke_9304.htm

Fabbrini, G., Barbanti, P., Aurilia, C., et al (2002) Donepezil in the treatment of hallucinations and delusions in Parkinson's disease. Neurological Science, 23, 41-43.

Fergusson, E. \& Howard, R. (2000) Donepezil for the treatment of psychosis in dementia with Lewy bodies. International Journal of Geriatric Psychiatry, 15, 280-281.

Finkel, S. I. (2004) Effects of rivastigmine on behavioral and psychological symptoms of dementia in Alzheimer's disease. Clinical Therapeutics, 26, 980-990.

Förstl, H., Burns, A., Levy, R., et al (1994) Neuropathological correlates of psychotic phenomena in confirmed Alzheimer's disease. British Journal of Psychiatry, 165, 5359 .

Fowler, D., Garety, P. \& Kuipers, E. (1995) Cognitive behavioural therapy for psychosis: theory and practice. Chichester: John Wiley \& Sons.

Frost, J. B. (1969) Paraphrenia and paranoid schizophrenia. Psychiatrica Clinica, 3, 129-138.

Garety, P., Kuipers, E., Fowler, D., et al (1994) Cognitivebehavioural therapy for drug resistant psychosis. British Journal of Psychology, 67, 259-271.

Giladi, N., Treves, T. A., Paleacu, D., et al (2000) Risk factors for dementia, depression and psychosis in long-standing Parkinson's disease. Journal of Neural Transmission, 107, 59-71.

Gilley, D. W., Wilson, R. S., Beckett, L. A., et al (1997) Psychotic symptoms and physically aggressive behaviour in Alzheimer's disease. Journal of the American Geriatrics Society, 45, 1074-1079.

Hall, L. \& Hare, J., (1997) Video respite for cognitively impaired persons in nursing homes. American Journal of Alzheimer's Disease and Other Dementias, 12, 117-121.

Herrmann, N., Mamdani, M. \& Lanctot, K. L. (2004) Atypical antipsychotics and risk of cerebrovascular accidents. American Journal of Psychiatry, 161, 1113-1115.

Hirose, T., Uwahodo, Y., Yamada, S., et al (2004) Mechanism of action of aripiprazole predicts clinical efficacy and a favourable side-effect profile. Journal of Psychopharmacology, 18, 375-383.

Hoeh, N., Gyalai, L., Weistraub, D., et al (2003) Pharmacological management of psychosis in the elderly. A critical review. Journal of Geriatric Psychiatry and Neurology, 16, 213-218.

Holmes, C., Fortenza, O., Powell, J., et al (1997) Do neuroleptic drugs hasten cognitive decline in dementia? Carriers of apolipoprotein E epsilon 4 allele seem particularly susceptible to their effects. BMJ, 10, 1411; author reply 1412.

Howard, R. (2002) Late-onset schizophrenia and very lateonset schizophrenia-like psychosis. In Psychiatry in the Elderly (eds R. Jacoby \& C. Oppenheimer), pp. 744-761. Oxford: Oxford University Press.

Howard, R. \& Levy, R. (1992) Which factors affect treatment response in late paraphrenia? International Journal of Geriatric Psychiatry, 7, 667-672.

Howard, R., Rabins, P. V., Seeman, M. V., et al (2000) International Late-onset Schizophrenia Group. Late-onset schizophrenia and very-late-onset schizophrenia-like psychosis: an international consensus. American Journal of Psychiatry, 157, 172-178.

Jaskiw, G. E., Thyrum, P. T., Fuller, M. A., et al (2004) Pharmacokinetics of quetiapine in elderly patients with select psychotic disorders. Clinical Pharmacokinetics, 43 $1025-1035$.

Jeste, D. V. (2004) Tardive dyskinesia rates with atypical antipsychotics in older adults. Journal of Clinical Psychiatry, 65 (suppl. 9), 21-24.

Jeste, D. V. \& Finkel, S. I. (2000) Psychosis of Alzheimer's disease and related dementias. Diagnostic criteria for a distinct syndrome. American Journal of Geriatric Psychiatry, $8,29-34$.

Jeste, D. V. \& Twamley, E. W. (2003) Understanding and managing psychosis in late life. Psychiatric Times, XX (3). 
Jeste, D. V., Eastham, J. H., Lacro, J. P., et al (1996) Management of late-life psychosis. Journal of Clinical Psychiatry, $\mathbf{5 7}$ (suppl. 3), 39-45.

Kane, J. M. (1999) Prospective study of tardive dyskinesia in the elderly. In Syllabus and Proceedings of the 1999 American Psychiatric Association Annual Meeting (Abstract NO32C:79). Washington, DC: APA

Karim, S. \& Burns, A. (2003) The biology of psychosis in old people. Journal of Geriatric Psychiatry and Neurology, 16, 207-212.

Katz, I. R, Jeste, D. V., Mintzer, J. E., et al (1999) Comparison of risperidone and placebo for psychosis and behavioural disturbances associated with dementia: a randomised double blind trial. Risperidone Study Group. Journal of Clinical Psychiatry, 60, 107-115.

Kay, D. W. K. \& Roth, M. (1961) Environment and hereditary factors in the schizophrenias of old age ("late paraphrenia") and their bearing on the general problem of causation in schizophrenia. Journal of Mental Science, $\mathbf{1 0 7}$ 649-686

Kidder, S. W. (2003) Psychosis in the elderly - whose delusion is it? Geriatric Times, 4 (2), 25-26.

Kopelowicz, A. \& Liberman, R. P. (1998) Psychosocial treatments for schizophrenia. In A Guide to Treatments that Work (eds P. E. Nathan \& J. M. Gorman), pp. 190-211. New York: Oxford University Press.

Kuzuhara, S. (2001) Drug-induced psychotic symptoms in Parkinson's disease. Problems, management and dilemma. Journal of Neurology, 248 (suppl. 3), III28-III31.

Levy, M. L., Cummings, J. L., Fairbanks, L. A., et al (1999) Apolipoprotein E genotype and non-cognitive symptoms in Alzheimer's disease. Biological Psychiatry, 165, 53-59.

Lisa, T., Zorrilla, E. \& Jeste, D. (2002) Late-life psychotic disorders: nosology and classification. In Principles and Practices of Geriatric Psychiatry (2nd edn) (eds J. R. M. Copeland, M. T. Abou-Saleh \& D. Blazer), pp. 493-496. Chichester: John Wiley \& Sons.

Madhusoodanan, S., Suresh, P. \& Brenner, R. (1999) Experience with the atypical anti-psychotics risperidone and olanzapine in the elderly. Annals of Clinical Psychiatry, 11, 113-118.

Madhusoodanan, S., Brenner, R., Gupta, S., et al (2004) Clinical experience with aripiprazole treatment in ten elderly patients with schizophrenia or schizoaffective disorder: retrospective case studies. CNS Spectrum, 9, 862867.

Margallo-Lana, M., Swann, A., O'Brien, J., et al (2001) Prevalence and pharmacological management of behavioural and psychological symptoms amongst dementia sufferers living in care environments. International Journal of Geriatric Psychiatry, 16, 39-44

McKeith, I. G., Fairbairn, A., Perry, R., et al (1992) Neuroleptic sensitivity in patients with senile dementia of Lewy body type. $B M J, 305,673-678$

McKeith, I. G., Fairbairn, A. F., Bothwell, R. A., et al (1994) An evaluation of the predictive validity and inter-rater reliability of clinical diagnostic criteria for senile dementia of Lewy body type. Neurology, 44, 872-877.

McKeith, I. G., Galasko, D., Kosaka, K., et al (1996) Consensus guidelines for the clinical and pathological diagnosis of dementia with Lewy bodies (DLB): report of the Consortium on DLB International Workshop. Neurology, 47, 1113-1124.

McKeith, I. G., Del Ser, T., Spano, P., et al (2000) Efficacy of rivastigmine in dementia with Lewy bodies: a randomised, double-blind, placebo-controlled international study. Lancet, 356, 2031-2036.

McKitrick, L., Camo, C. \& Black, F. (1992) Prospective memory intervention in Alzheimer's disease. Journal of Gerontology, 47, P337-P343.

McManus, D. Q., Arvanitis, L. A. \& Kowalcyk, B. B. (1999) Quetiapine, a novel antipsychotic: experience in elderly patients with psychotic disorders. Journal of Clinical Psychiatry, 60, 292-298.

McQuaid, J. R., Granhoolm E., McClure, F. S., et al (2000) Development of an integrated cognitive-behavioural and social skills training intervention for older people with schizophrenia. Journal of Psychotherapy Practice and Research, 9, 149-156.

McShane, R., Keene, J., Gedling, K., et al (1997) Do neuroleptic drugs hasten cognitive decline in dementia? Prospective study with necropsy follow up. BMJ, 314, 266-270.

Mintzer, J. \& Targum, S. D. (2003) Psychosis in elderly patients: classification and pharmacotherapy. Journal of Geriatric Psychiatry and Neurology, 16, 199-206.

Mowat, D., Fowlie, D. \& MacEwan, T. (2004) CSM warning on atypical psychotics and stroke may be detrimental for dementia. BMJ, 328, 1262.

Ondo, W. G., Levy, J. K., Vuong, K. D., et al (2002) Olanzapine treatment for dopaminergic-induced hallucinations. Movement Disorders, 17, 1031-1035.

Östling, S. \& Skoog, I. (2002) Psychotic symptoms and paranoid ideation in a non-demented population-based sample of the very old. Archives of General Psychiatry, 59, 53-59.

Overshott, R., Byrne, J. \& Burns, A. (2004) Nonpharmacological and pharmacological interventions for symptoms in Alzheimer's disease. Expert Review of Neurotherapeutics, 4, 809-821.

Palmer, B. W., McClure, F. S. \& Jeste, D. V. (2001) Schizophrenia in late life: findings challenge traditional concepts. Harvard Review of Psychiatry, 9, 51-58.

Post, F. (1966) Persistent Persecutory States of the Elderly. Oxford: Pergamon Press.

Royal College of Psychiatrists, Royal College of General Practitioners, British Geriatrics Society, et al (2004) Guidance for the Management of Behavioural and Psychiatric Symptoms in Dementia and the Treatment of Psychosis in People with History of Stroke/TIA Following CSM Restriction on Risperidone and Olanzapine. London: Faculty of the Psychiatry of Old Age, Royal College of Psychiatrists. http: //www.rcpsych.ac.uk/college/faculty / oap / professional/guidance_summary.htm

Sajatovic, M., Perez, D., Brescan, D., et al (1998) Olanzapine therapy in elderly patients with schizophrenia. Psychopharmacology Bulletin, 34, 819-823.

Schneider, L. S. (1996) Meta-analysis of controlled pharmacologic trials. International Psychogeriatrics, 8 (suppl. 3), 375-379; discussion 381-382.

Schneider, L. S., Olin, J. T., Doody, R. S., et al (1997) Validity and reliability of the Alzheimer's Disease Cooperative Study - Clinical Global Impression of Change. The Alzheimer's Disease Cooperative Study. Alzheimer Disease and Associated Disorders, 11 (suppl. 2), S22-S32.

Steele, C., Rovner, B., Chase, G. A., (1990) Psychiatric symptoms and nursing home placement of patients with Alzheimer's disease. American Journal of Psychiatry, 147, 1049-1051.

Stern, Y., Tang, M. X., Albert, M. S., et al (1997) Predicting time to nursing home care and death in individuals with Alzheimer's disease. JAMA, 277, 806-812.

Street, J. S., Clark, W. S., Gannon, K. S., et al (2000) Olanzapine treatment of psychotic and behavioural symptoms in patients with Alzheimer disease in nursing care facilities: a double-blind, randomised, placebo-controlled trial. The HGEU Study Group. Archives of General Psychiatry, 57, 968-976.

Street, J. S., Clark, W. S., Kadam, D. L., et al (2001) Longterm efficacy of olanzapine in the control of psychotic and behavioral symptoms in nursing home patients with Alzheimer's dementia. International Journal of Geriatric Psychiatry, 16 (suppl. 1), S62-S70.

Targum, S. D. (2001) Treating psychotic symptoms in elderly patients. Primary Care Companion to the Journal of Clinical Psychiatry, 3, 156-163.

Targum, S. D. \& Abbott, J. L. (1999) Pyschosis in the elderly: a spectrum of disorders. Journal of Clinical Psychiatry, 60 (suppl. 8), 4-10.

Tariot, P. N. (1995) Behaviour Rating Scale for Dementia American Journal of Psychiatry, 152, 1349-1357.

Tariot, P. N., Salzman, C., Yeung, P. P., et al (2000) Longterm use of quetiapine in elderly patients with psychotic disorders. Clinical Therapeutics, 22, 1068-1084. 
Tsuang, M. M., Lu, L. M., Stotsky, B. A., et al (1971) Haloperidol versus thioridazine for hospitalised psychogeriatric patients: double blind study. Journal of the American Geriatrics Society, 19, 593-600.

Tune, L. E. \& Salzman, C. (2003) Schizophrenia in late life. Psychiatric Clinics of North America, 26, 103-113.

Walker, Z., Grace, J., Overshot, R., et al (1999) Olanzapine in dementia with Lewy bodies: a clinical study. International Journal of Geriatric Psychiatry, 14, 459-466.

Wolters, E. C. (2001) Intrinsic and extrinsic psychosis in Parkinson's disease. Journal of Neurology, 248 (suppl. 3), III22-III27.

Wolters, E. C. \& Berendse, H. W. (2001) Management of psychosis in Parkinson's disease. Current Opinion in Neurology, 14, 499-504.

Wood, K. A., Harris, J., Morreale, A., et al (1988) Druginduced psychosis and depression in the elderly. Psychiatric Clinics of North America, 11, 167-193.

World Health Organization (1992) The ICD-10 Classification of Mental and Behavioural Disorders. Clinical Descriptions and Diagnostic Guidelines. Geneva: WHO.

Wynn, Z. J. \& Cummings, J. L. (2004) Cholinesterase inhibitor therapies and neuropsychiatric manifestations of Alzheimer's disease. Dementia and Geriatric Cognitive Disorders, 17, 100-1008.

Yeung, P. P., Tariot, P. N., Schneider, L. S., et al (2000) Quetiapine for elderly patients with psychotic disorders. Psychiatric Annals, 30, 197-203.

Zarit, S. H., Todd, P. A. \& Zarit, J. M. (1986) Subjective burden of husbands and wives as caregivers: a longitudinal study. Gerontologist, 26, 260-266.

Zayas, E. M. \& Grossberg, G. T. (1998) The treatment of psychosis in late life. Journal of Clinical Psychiatry, 59 (suppl. 1), 5-12.

Zayas, E. M. \& Grossberg, G. T. (2002) Treatment of lateonset psychotic disorders. In Principles and Practices of Geriatric Psychiatry (2nd edn) (eds J. R. M. Copeland, M. T. Abou-Saleh \& D. Blazer), pp. 511-525. Chichester: John Wiley \& Sons.

\section{MCQs}

1 Psychotic symptoms in elderly people:

a are diagnostic of schizophrenia

b can be seen in dementia

c are always secondary to a medical illness

d carry a higher caregiver burden

e can result in neglect of patients.

2 Schizophrenia in elderly people :

a always presents with visual hallucinations

b requires $\mathrm{CT}$ brain scan for diagnosis

c can be diagnosed at any age

d should be treated by isolating the patient

e can have associated cognitive deficits.

3 In people with Alzheimer's disease:

a psychotic symptoms are rare

b delusions are the most common psychotic symptoms

c antipsychotics are not effective for psychotic symptoms

d psychotic symptoms can be managed without medication

e psychotic symptoms can be secondary to cognitive deficits.

4 Regarding psychotic symptoms in Lewy body dementia:

a visual hallucinations are the most common feature

b antipsychotics are the first line of treatment

c can be induced by anti-Parkinsonian medication

d have been shown to improve with cholinesterase inhibitors

e rivastigmine is licensed in UK for their treatment.

5 Regarding use of antipsychotics in elderly people with schizophrenia:

a the risk of developing tardive dyskinesia is higher with conventional antipsychotics

b can increase the risk of falls

c depot antipsychotics are contraindicated

d clozapine is the first-line choice

e the usual starting dose is half the adult dose.

\section{MCQ answers}

\begin{tabular}{|c|c|c|c|c|}
\hline 1 & 2 & 3 & 4 & 5 \\
\hline a $\mathrm{F}$ & a $\mathrm{F}$ & a $\mathrm{F}$ & a $\mathrm{T}$ & a \\
\hline b $\mathrm{T}$ & b F & $\mathrm{b} T$ & b F & b \\
\hline $\mathrm{F}$ & с $\mathrm{T}$ & c F & с $\mathrm{T}$ & C \\
\hline $\mathrm{T}$ & $\mathrm{d} F$ & $\mathrm{~d} \mathrm{~T}$ & $\mathrm{~d} \mathrm{~T}$ & \\
\hline $\mathrm{T}$ & e $\mathrm{T}$ & e $\mathrm{T}$ & e $\mathrm{F}$ & \\
\hline
\end{tabular}

